\title{
Autocatalytic Polymerization Generates Persistent Random Walk of Crawling Cells
}

\author{
R. Sambeth ${ }^{1}$ and A. Baumgaertner ${ }^{1,2}$ \\ ${ }^{1}$ Forum Modellierung, Forschungszentrum Jülich, D-52425 Jülich, Germany \\ ${ }^{2}$ Institut für Festkörperforschung, Forschungszentrum Jülich, D-52425 Jülich, Germany
}

(Received 11 January 2001)

\begin{abstract}
The autocatalytic polymerization kinetics of the cytoskeletal actin network provides the basic mechanism for a persistent random walk of a crawling cell. It is shown that network remodeling by branching processes near the cell membrane is essential for the bimodal spatial stability of the network which induces a spontaneous breaking of isotropic cell motion. Details of the phenomena are analyzed using a simple polymerization model studied by analytical and simulation methods.
\end{abstract}

DOI: 10.1103/PhysRevLett.86.5196

The amoeboid crawling of animal cells like fibroblasts and keratocytes or the advancing neural growth cone has been the subject of intensive experimental [1-5] and theoretical [6-11] studies. It is now commonly accepted that the continuous remodeling of the actin cytoskeleton of a cell provides the main contribution $[5,7,8]$ to the driving force which leads to cell motility. In particular, it has been suggested that the polymerization of monomeric $G$-actin to filamentous $F$-actin oriented essentially perpendicular to the cell membrane provides the necessary motor for membrane protrusion and hence motility. However, the phenomenon of cell locomotion cannot be explained solely based on the $G$ - to $F$-actin transition. In particular, the reasons for the observed characteristic traces of cell migration exhibiting a pattern similar to a random walk with a certain persistency $[12,13]$ has remained obscure. In the present Letter, we show that random walk characteristics and persistency can be the results of the special bifurcated structure of the actin cytoskeleton $[13,14]$ and of its inherent autocatalytic polymerization kinetics.

The model which is considered in the present work corresponds to a special cell fragment, the cytoplast, which has been studied experimentally [13,15-17] in order to monitor cell locomotion generated solely by the actin cytoskeleton. The cytoplast is a fragmentary keratocyte where the nucleus has been removed. This fragment is either at rest or exhibits a directional and persistent motility. The persistency of the motion can be described as a sequence of linked vectors which differs from that of a true random walk in that the angles between successive vectors are correlated.

During the motile phase the cell is spatially deformed to a half-moon shape and the leading edge is pushed forward by a dense actin network, whereas at the rear the network is much less dense. In the resting phase the cytoplast is spherically symmetric and the network is continuously distributed along the cell membrane. The transition between the two phases occurs spontaneously but can also be induced by mechanical stimuli. Clearly a positive feedback mechanism must be present that can amplify density fluctuations of the symmetric resting state and maintain the asymmetric motile structure for a certain time. It has been
PACS numbers: 87.17. $-\mathrm{d}, 05.40 .-\mathrm{a}, 82.35 .-\mathrm{x}$

suggested recently [13] that the feedback can be attributed to the contractivity of the motor protein myosin II. We propose here a different mechanism in which the asymmetry is induced and maintained by the autocatalytic properties of the polymerizing actin network.

Actin filaments are chemically linked into networks which have some peculiar and important structural properties: they are polymer networks with a treelike structure $[14,18]$. The polymerization consists essentially of two processes: preexisting actin filaments grow linearly at asymmetric rates with respect to both ends [12] and daughter branches grow out of mother filaments at a bifurcation angle of about $70^{\circ}$. The branching process starts as soon as the cytosolic Arp2/3 protein [14] becomes attached to the mother filament. The important point is that the cellular distribution of $\operatorname{Arp} 2 / 3$ is not homogeneous but is localized near the cell membrane. Therefore, network formation is dominant near the cell membrane, whereas depolymerization and linear growth dominates at the center of the cell.

In contrast to previous works $[7,8,11,19]$ where externally guided linear growth has been employed in order to obtain cell migration, the present work shows that in the case of absence of external guidance network polymerization is an inevitable prerequisite for cell locomotion. In particular, it is shown that the autocatalytic properties of network polymerization near the cell membrane represent the predominant mechanism which spontaneously breaks the symmetry of motion and induces a persistent random walk.

The paper is organized in two sections. In the first part we report on computer simulations of a cytoplast model, and in the second part we present a simple mathematical model which describes the observed phenomena. In the following part of this Letter we report on computer simulations of a cell model representing essential features of a cytoplast.

A typical snapshot is depicted in Fig. 1. The model represents a horizontal section cut from a large twodimensional cell model where the cell membrane is represented by a large flexible polymer ring. The cut has been performed along the upper and lower broken 


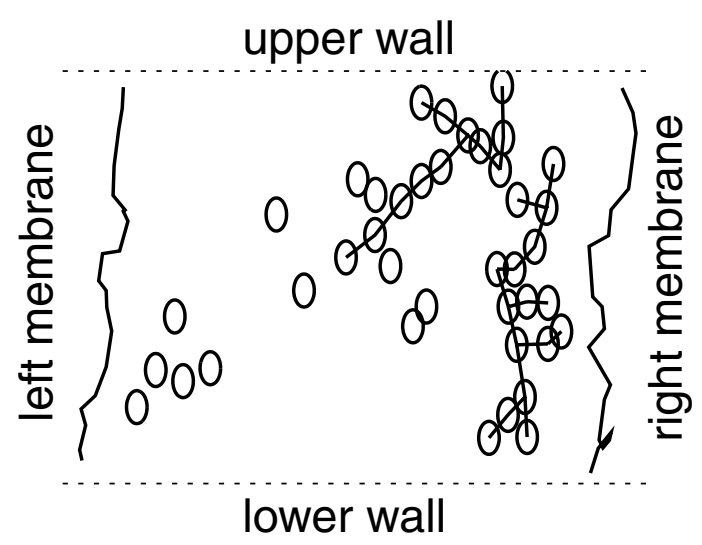

FIG. 1. Snapshot of the computer model, explained in the text.

lines as shown in Fig. 1. Henceforth the broken lines are called the upper and lower walls because they are impenetrable for molecules. The remaining parts of the cell membrane are depicted in Fig. 1 and are denoted by left membrane and right membrane. The main reason for considering such a cell section rather than the complete cell is clarity, because this type of model is effectively one dimensional in the $x$ direction which makes it much easier to characterize the persistency of cell motion.

In order to maintain the integrity of such a cell section, three constraints have been introduced. The distance between the walls is $\ell=$ const. The ends of the membranes are attached by harmonic springs to the walls. In addition, in order to prevent uncontrolled lateral expansion of the cell section, the distances between the two membranes are restricted by harmonic forces, each acting along the upper and lower walls between the ends of the membranes. It should be noted that these constraints on the membranes do not change qualitatively the basic conclusions of the present study.

The cell section contains a certain number of actin molecules which are depicted in Fig. 1 by open circles. Some of the actin molecules are connected to filamentous networks. The actin networks are assumed to be tightly coupled to an underlying substrate by external harmonic forces in order to break momentum conservation of the entire cell [11]. Hence, the networks are at rest on the average with respect to an external coordinate system in the $x y$ plane. The interactions between actin molecules and cell membrane and walls are repulsive. Consecutive actin molecules in a single network are connected by harmonic forces. Elastic bending forces control the stiffness of the filaments. Single actin molecules (monomers) diffuse freely inside the cell. All particles, including actin monomers and membrane segments, move in a fluid with temperature $T$ and their motion is described by the Langevin equation. All particles are equally sized with radius $a=0.08 \ell$, and their diffusion coefficients are $D$ which define the time scale $\tau=a^{2} / D$.
Each network (as depicted in Fig. 1) can grow by addition polymerization of actin monomers at the ends. The growth rate is purely diffusion limited, i.e., association takes place as soon as an actin monomer comes into contact with one of the ends of the networks. This type of polymerization process represents a continuation growth of existing filaments. In addition, there exists a second type of polymerization process, branching, which takes place predominantly near the cell membrane. Within a distance $d=0.2 \ell$ from one of the two membranes, actin molecules of the network may be decorated with an Arp2/3 molecule, which enables the actin monomer to be a branching point for daughter filaments. This branching mechanism is introduced in accordance with experimental observations which indicate that association of cytosolic Arp2/3 proteins with actin molecules induces branching of actin filaments [14]. Arp $2 / 3$ proteins are not explicitly simulated but are assumed to be continuously generated by some biochemical processes near the intracellular cell membrane [14].

Networks can shrink by depolymerization processes. The dissociation of actin molecules from filaments in vitro is asymmetric in the sense that the two ends of a filament behave differently [12]. The "polarity" of actin molecules leads to a fast-growing (plus) end with a low dissociation rate and a slower-growing (minus) end with a high dissociation rate. In the present model we define, for simplicity, the dissociation rate at the plus end to be zero, whereas at a free minus end monomers are depolymerized with an infinite rate, if they are not decorated by an Arp2/3. Branching points are dissociated with rate $k_{b}$. In order to avoid complete depolymerization of all networks a rate $k_{n}$ was introduced for de novo nucleation of actin filaments.

One simulation step consists of the calculation of all forces, the displacement of each particle according to its Langevin equation [20], the evaluation of the excluded volume forces by an elastic scattering approach $[21,22]$ and the calculation of all polymerization, depolymerization, and branching reactions. The trajectory of a typical simulation is given in Fig. 2: the large solid curve represents the $x$ displacements $x_{M}(t)$ of the center of the cell, i.e., the center of mass of the two membranes, as a function of time $t / \tau$. Displacements $x_{M}(t)$ and distribution $p_{F}(x, t)$ clearly exhibit a correlation: motion in one of the two $x$ directions is in concomitance with a strongly asymmetric distribution of $F$-actin towards the same direction. Obviously, the interpretation is that asymmetric growth and distribution of $F$-actin push the membrane in one direction as long as the diffusion-limited supply of new $G$-actin monomers near one cell membrane is possible.

On the other hand, the stochasticity of this process may lead sometimes to an amplified growth near the opposite membrane which may change then the direction of migration. Therefore, on the average, the distribution of $F$-actin is bimodal. This is shown by the two-dimensional distribution $p f(x, y)$ in the upper right inset in Fig. 2. The important point is that this bimodal distribution is strongly 


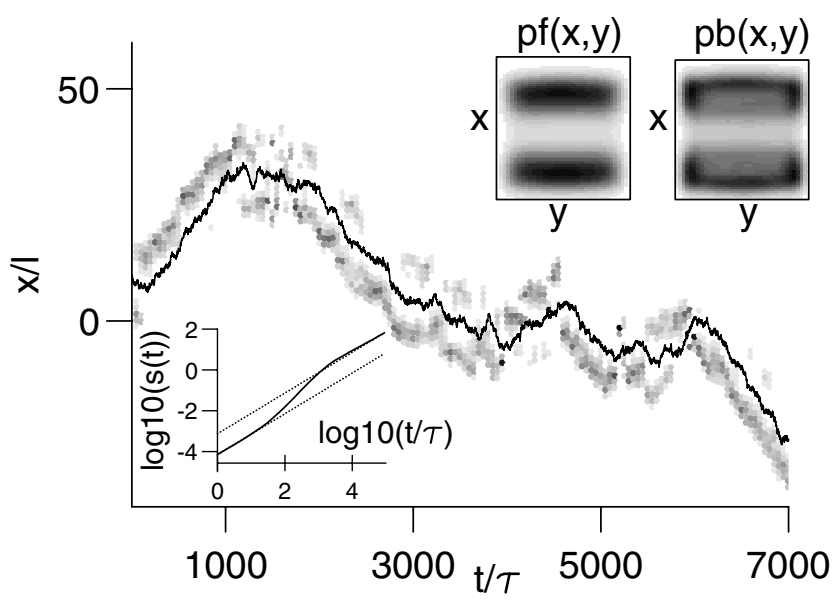

FIG. 2. Trajectory, $F$-actin distribution (upper right insets) and mean square displacement (lower left inset) of a simulation. The shaded areas above and below the trajectory represent the number distribution $p_{F}(x, t)$ of actin molecules bound into networks ( $F$-actin). Black, grey, and white shadings correspond to high, intermediate, and low probability, respectively. Averaging in the $y$ direction has been performed.

correlated to the distribution of the plus ends of the networks which is shown by the probability $p b(x, y)$ in the upper right inset in Fig. 2. The distribution indicates that the plus ends are found with higher probability at the boundary of the cell than anywhere else. Since the number of plus ends are closely related to the number of branching points, this indicates that the stability of a certain asymmetric distribution of $F$-actin within the cell must be related to the statistical properties of the branching processes, and not to chemical processes. This is an important point and explains experimental observations [5] similar to the distribution $p b(x, y)$. More details of the statistical properties of branching processes are discussed below in the theoretical part of this Letter.

Long runs of simulations provide the complete picture of a persistent random walk which can be characterized by the mean square displacements of the center of mass $X$ of the entire cell $s(t) \equiv\left\langle[X(t)-X(0)]^{2}\right\rangle$ as a function of time. This is shown in the lower left inset in Fig. 2. At short times, $\log 10(t)<2$, the center of mass exhibits Brownian motion according to $s(t)=D_{1} t$. At intermediate times, $2<\log 10(t)<3$, a rectified motion is observed, $s(t)=$ $(v t)^{2}$, where $v$ is the average velocity. And finally for $\log 10(t)>3$ the cell performs a persistent random walk, $s(t)=D_{2} t$, where $D_{2} \approx 6.5 D_{1}$.

The simulation results as described above can be understood and described by the following mathematical model which provides an explanation for the stability and amplification of network inhomogeneity. Consider a closed system of $N$ actin monomers in a volume $V$ proportional to $N$ that can polymerize into filaments. This process can be described as a one-step process [23] by the following master equation for the probability $p(n)$ to find $n$ particles polymerized:

$$
\frac{d p(n)}{d t}=\left(1-\delta_{n 1}\right) j(n)-\left(1-\delta_{n N}\right) j(n+1),
$$

where $j(n)=g(n-1) p(n-1)-r(n) p(n)$ is the current, $g(n)=k^{+} n^{\alpha}(N-n) / N$ is the polymerization rate, and $r(n)=k^{-} n^{\beta}$ is the depolymerization rate. The use of the Kronecker $\delta$ ensures reflecting boundaries for $n=$ $1, N$. For $\alpha=0$ the number of polymerization sites remains constant, whereas for $\alpha>0$ the number of sites increases with the number of polymerized units. This situation corresponds to a branching network, where a newly added monomer increases the number of ends by one. Therefore, setting $\alpha>0$ effectively accounts for the action of Arp2/3. Similarly, the depolymerization rate $r(n)$ corresponds to a fixed number of depolymerization sites for $\beta=0$ whereas for $\beta>0$ the number of such sites increases leading to the disintegration of a given network into separate networks.

At values $\theta=k^{-} / k^{+}<1$ and $\gamma=\alpha-\beta>0$ the stationary probability distribution [23]

$$
p^{s}(n)=C \frac{[(n-1) !]^{\alpha}(N-1) !}{(\theta N)^{n-1}(n !)^{\beta}(N-n) !}
$$

is peaked around a value $n_{p} \gg 0$ which can be found approximately by solving the deterministic equation corresponding to Eq. (1), $d n_{p} / d t=\left(N-n_{p}\right) n_{p}^{\gamma}-N \theta=0$, which is shown in Fig. 3.

This polymerization model is a generalization of the autocatalytic reaction $X+Y \rightarrow 2 X, \quad X \rightarrow Y$ which corresponds to $\alpha=\beta=1$ [24], and hence we call the process described by Eq. (1) as an autocatalytic polymerization process.

Now assume that there are two spatially separated sites where polymerization can occur. Consequently a monomer can polymerize in either region $A$ or region $B$. Then Eq. (1)

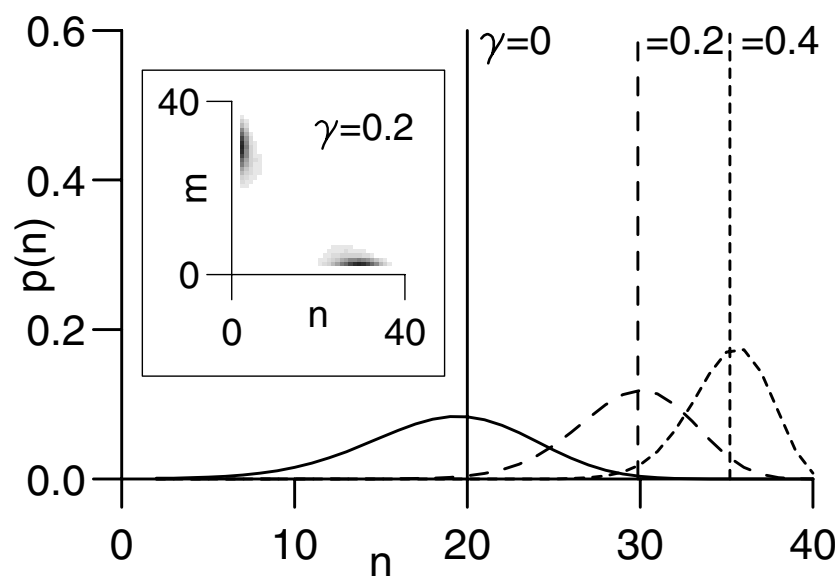

FIG. 3. $p(n)$ as defined in the text, centered around the deterministic mean value. Inset: $p(n, m)$ as density plot, black corresponds to high probability, and white to low probability. 
has to be replaced by

$$
\frac{d p(n, m)}{d t}=j^{+}-j^{-},
$$

where $j^{+}=\left(1-\delta_{n 1}\right) j_{A}(n, m)+\left(1-\delta_{m 1}\right) j_{B}(n, m)$ and $j^{-}=\left(1-\delta_{n N}\right) j_{A}(n+1, m)+\left(1-\delta_{m N}\right) j_{B}(n, m+1)$. Equation (2) describes the two-dimensional one-step process for the probability $p(n, m)$ to find $n$ polymerized particles in region $A$ and $m$ particles in region $B$. The currents are

$$
j_{A}(n, m)=g_{A}(n-1, m) p(n-1, m)-r_{A}(n, m) p(n, m)
$$

and

$j_{B}(n, m)=g_{B}(n, m-1) p(n, m-1)-r_{B}(n, m) p(n, m)$.

The rates are defined as $g_{A}(n, m)=g_{B}(m, n)=$ $k^{+} n^{\alpha}[N-(n+m)] / N \quad$ and $\quad r_{A}(n, m)=r_{B}(m, n)=$ $k^{-} n^{\beta}$. For the same $\theta$ as defined above, the stationary solution of Eq. (2) is

$$
p^{s}(n, m)=C^{\prime} \frac{[(n-1) !(m-1) !]^{\alpha}(N-1) !}{(\theta N)^{n+m-2}(n ! m !)^{\beta}[N-(n+m)] !} .
$$

This distribution is bimodal, with one peak at $n=n_{p}$, $m \approx 1$ and the second at $m=n_{p}, n \approx 1$; see the inset in Fig. 3. At $\gamma=0.2$ the two peaks do not overlap, i.e., almost all monomers are in either region $A$ or region $B$. The polarization $\Pi=n-m$ has the average value $\langle\Pi\rangle=0$, but in the steady state jumps between the two metastable values $\Pi \approx \pm n_{p}$. This is a direct consequence of $\gamma>0$, which is due to the branching character of the filament networks. The average frequency of jumps can be found by transforming

$$
(n, m) \rightarrow k(n, m)=\frac{1}{2}(n+m-2)(n+m-1)+n
$$

and writing Eq. (2) in a matrix form for probabilities $p(k)$. Then the jump frequency is given by the largest eigenvalue of the stochastic matrix that is smaller than zero. It can be shown numerically that for $\gamma=0$ the transition frequency is of the same magnitude as $k^{+}+k^{-}$and that for $\gamma>0$ the magnitude increases by several orders resulting in two metastable states. The transition between those states is induced by fluctuations, and the most probable pathway can be found numerically and depends on the choice of parameters.

The values of $\alpha$ and $\beta$ in the computer simulation described above are not set externally but can be estimated from the simulation results. For the parameters of Fig. 1 the values are $\alpha=0.97$ and $\beta=0.72$, so that indeed $\gamma=\alpha-\beta>0.2$ which provides a bimodal distribution of $F$-actin: networks are found at either the left or the right of the model cell.

To summarize, we have shown that the polymerization of a branching actin filament network leads to an autocatalytic, nonlinear increase of free plus ends. This in turn accounts for the bistable behavior of the one-dimensional cell model: almost all $F$-actin is found either at one membrane or the other, but not in between. Consequently the model cell performs the persistent random motion that is known from crawling animal cells in vitro.

[1] H. Gruler and B. D. Bültmann, Blood Cells 10, 61 (1984).

[2] J. A. Theriot and T. J. Mitchison, Nature (London) 352, 126 (1991).

[3] J. Lee, A. Ishihara, J. A. Theriot, and K. Jacobson, Nature (London) 362, 167 (1993).

[4] C.-H. Lin and P. Forscher, Neuron 14, 763 (1995).

[5] T. M. Svitkina, A. B. Verkhovsky, K. M. McQuade, and G. G. Borisy, J. Cell Biol. 139, 397 (1997).

[6] H. Gruler, Blood Cells 19, 91 (1993).

[7] C. S. Peskin, G. M. Odell, and G. F. Oster, Biophys. J. 65, 316 (1993).

[8] A. Mogilner and G. F. Oster, Biophys. J. 71, 3030 (1996).

[9] D. C. Bottino and L. J. Fauci, Eur. Biophys. J. 27, 532 (1998).

[10] W. Alt and M. Dembo, Math. Biosci. 156, 207 (1999).

[11] R. Sambeth and A. Baumgaertner (to be published).

[12] D. Bray, Cell Movements (Garland, New York, 1992).

[13] A. B. Verkhovsky, T. M. Svitkina, and G. G. Borisy, Curr. Biol. 9, 11 (1999).

[14] R. D. Mullins, J. A. Heuser, and T. D. Pollard, Proc. Natl. Acad. Sci. U.S.A. 95, 6181 (1998).

[15] G. Albrecht-Buehler, Proc. Natl. Acad. Sci. U.S.A. 77, 6639 (1980).

[16] S.E. Malawista and A. de Boisfleury-Chevance, J. Cell Biol. 95, 960 (1982).

[17] U. Euteneuer and M. Schliwa, Nature (London) 310, 58 (1984).

[18] T. M. Svitkina and G. G. Borisy, J. Cell Biol. 145, 1009 (1999).

[19] L. Edelstein-Keshet, Eur. Biophys. J. 27, 521 (1998).

[20] W. F. van Gunsteren and H. J. C. Berendsen, Mol. Phys. 45, 637 (1982).

[21] P. Strating, Phys. Rev. E 59, 2175 (1999).

[22] R. Sambeth and A. Baumgaertner, Physica (Amsterdam) 271A, 48 (1999).

[23] N. G. van Kampen, Stochastic Processes in Physics and Chemistry (North-Holland, Amsterdam, 1992).

[24] I. Oppenheim, K. E. Shuler, and G. E. Weiss, Physica (Amsterdam) 88A, 191 (1977). 